\title{
Review of: "Detección de Aedes (Stegomyia) albopictus (Skuse) en ovitrampas en Mérida, México"
}

\author{
Rafael Piovezan ${ }^{1}$ \\ 1 São Paulo State University (UNESP)
}

Potential competing interests: The author(s) declared that no potential competing interests exist.

Review Article: “Dtección de Aedes (Stegomyia) albopictus (Skuse) en ovitrampas em Mérida, México” de González-Olvera et al. - Biomédica 2021; 41:153-60

The article provides a important information about the spatial dynamics of Aedes (Stegomyia) aegypti (Linnaeus, 1762) and Aedes albopictus in Mérida, Mexico. These two species are extremely important for the epidemiology of arbovirus transmission in the world. An analysis of this concept demonstrates that it is estimated that about 2,5 billion people are at risk of acquiring the Dengue virus infections worldwide (LiuHelmersson et al., 2014). In addition to Dengue virus, mosquitoes can carry almost 4000 arboviruses (Powel, 2018), among them the Mayaro, Venezuelan equine encephalitis, Eastern equine encephalitis, Chikungunya, yellow fever, Rocio, Saint Louis encephalitis, West Nile; and Oropouche (Figueiredo, 2007). Moreover, malaria and bancroftian filariasis are mosquito-borne diseases that affect populations inhabiting tropical and subtropical areas (Marcondes and Ximenes, 2016).

The epidemiological importance of Aedes albopictus

Although, in Brazil, for example, Ae. albopictus has not been incriminated in the transmission of the Dengue virus, it possesses the proven competence of transmitting several arboviruses such as Dengue and Chikungunya (Gratz, 2004; Marcondes and Ximenes, 2015). Further, as regards dengue, this vector's ability has been proved by the epidemics that have occurred in Japan, Indonesia, the Seychelles, Thailand and Malaysia (Gratz, 2004), in Hawaii (Effler et al., 2005), Gabon (Paupy et al., 2010) and in Europe (Field et al., 2010; La Ruche et al., 2010). The Chikungunya virus has also been transmitted by Ae. albopictus, as has recently been reported in Europe, Africa and the Indian Ocean (Reiter et al., 2006; Bonilauri et al., 2008; Pagès et al., 2009; Paupy et al., 2010).

Aedes albopictus, therefore, is an important species to be monitored, as well the constant monitoring of ecological relationships established in urban areas and transitional environments is essential. The interaction between the species Ae. aegypti and Ae. albopictus may be influenced by environmental factors. In situations of co-habitation, the characteristics of the scenery and environmental conditions may be determining factors in the success or failure of the species. Places with accentuated rural characteristics and the presence of vegetation favor the displacement and exclusion of Ae. aegypti by Ae. 
albopictus, while in urban environments or those subject to great anthropic influence, Ae. aegypti persists or dominates over Ae. albopictus (Lounibos et al., 2010). In situations in which the quantity of food available is reduced or even slightly limited, the interspecific larval competition is the main factor determining the abundance of these two mosquito species and it is Ae. albopictus which, under these circumstances, has the advantage over Ae. aegypti (Braks et al., 2004). However, under environmental conditions such as the low relative humidity and high temperatures which prevail in drier periods, the competition between the two species may be reduced seeing that Ae. albopictus is more sensitive to these variations, both in the egg and in the adult phases (Juliano et al., 2002).

\section{Surveillance of Culicidae: Ovitraps}

The use of oviposition traps for surveillance of Ae. aegypti and Ae. albopictus is a sensitive and safe way to help mosquito control (Braga et al., 2000; Bezerra et al., 2014). Likewise, measuring the impact of interventions is of fundamental importance for the local program administrators for the dengue control. The article of González-Olvera et al. (2021) presents the detection of Ae. albopictus through a network of ovitraps installed in Mexico to monitor Aedes spp. The conclusion is that the species coexists with Ae. aegypti, among others Culicidae, and the Ae. albopictus it was found in $32 \%$ of the sampled neighborhoods of Mérida, establishing that the data indicate the beginning of the invasion of Ae. albopictus in the city. The record of Ae. albopictus is important because, as mentioned above, this species, in addition to having epidemiological relevance in the transmission of various etiological agents that mabe cause diseases in the human population, has the special capacity to infest transitional environments between urban and rural/wild areas. This ecological capacity makes Ae. albopictus a vector between these environments, and this is important to be monitored.

The work collected information between October 21 and 31, 2019, with data sampled from 91 ovitraps randomly chosen. Certainly, expanding the sampling for a few years, for example, will allow the autors to establish the dynamics of Ae. albopictus in a spatiotemporal analysis. An approach to the landscape must also be made, in order to establish which are the conditions that eventually allowed the presence of the species (Figures 1 and 2). These analyzes will also be important to understand more clear the ecological relationships between Ae. aegypti and Ae. albopictus, for example.

As a way to improve the sampling, if the proposal is to better understand the dynamics of the species, larvitraps can be included as containers for surveying Culicidae larvae, in addition to expanding the number of collection traps, which would allow a spatial analysis more detailed, whose data would bring more information for the entomological monitoring of mosquitoes and for the establishment of public policies to control arboviruses.

The Mexican National Dengue Control Program integrated vector surveillance and control platform receives information from 250,000 ovitraps distributed throughout the country, however this information is only about Ae. aegypti. Considering this information, the work correctly points out the need to include data from Ae. albopictus, which would help to provide information on the infestation of the two species. In addition, 
as already mentioned, the use of traps to collect larvae could also provide more complete information regarding the species that use artificial breeding sites for their development.

The work by González-Olvera et al (2021) provides relevant information about the cohabitation of Ae. aegypti and Ae. albopictus in the city of Mérida, Mexico. The data collected and analyzed does not allow more complex analyzes of the abundance and richness of these two species, since they were collected only in October 2019, in a few traps. The authors are right to emphasize the importance of ovitraps in terms of being important tools for monitoring and monitoring Aedes spp. The inclusion of larvitraps can also help in richness analyses. In addition, providing more samples can help the spatiotemporal analyses.

Reconciling the landscape with efficient data collection mechanisms for entomological information on mosquitoes contributes considerably to public policies to control arboviruses in urban areas. This is a relevant point that was considered in the authors' conclusions. 